\title{
Circ-AFF2/miR-650/CNP axis promotes proliferation, inflammatory response, migration, and invasion of rheumatoid arthritis synovial fibroblasts
}

Wei Qu' ${ }^{1}$ L Ling Jiang ${ }^{2}$ and Guanhua $\mathrm{Hou}^{3^{*}}$

\begin{abstract}
Background: Circular RNAs (circRNAs) are associated with rheumatoid arthritis (RA) development. The purpose of this study is to explore the function and mechanism of circRNA fragile mental retardation 2 (circ-AFF2) in the processes of rheumatoid arthritis fibroblast-like synoviocytes (RAFLSs).

Methods: Circ-AFF2, microRNA (miR)-650, and 2',3'-cyclic nucleotide 3'-phosphodiesterase (CNP) levels were determined in synovial tissues of RA and RAFLSs by quantitative reverse transcription polymerase chain reaction or Western blotting. Cell proliferation, inflammatory response, apoptosis, caspase3 activity, migration, invasion, and epithelial-mesenchymal transition (EMT) were investigated using Cell Counting Kit-8 (CCK-8), enzyme-linked immunosorbent assay (ELISA), flow cytometry, Transwell, and Western blotting analyses. Dual-luciferase reporter, RNA immunoprecipitation (RIP), and pull-down assays were performed to assess the binding relationship.

Results: Circ-AFF2 expression level was enhanced in synovial tissues of RA and RAFLSs. Circ-AFF2 overexpression facilitated cell proliferation, inflammatory response, migration, invasion, and EMT and repressed apoptosis in RAFLSs. Circ-AFF2 downregulation played an opposite role. Circ-AFF2 targeted miR-650, and miR-650 downregulation reversed the effect of circ-AFF2 interference on RAFLS processes. CNP was targeted by miR-650, and circ-AFF2 increased CNP expression by regulating miR-650. MiR-650 overexpression constrained cell proliferation, inflammatory response, migration, invasion, and EMT and contributed to apoptosis by decreasing CNP in RAFLSs.

Conclusion: Circ-AFF2 promoted proliferation, inflammatory response, migration, and invasion of RAFLSs by modulating the miR-650/CNP axis.
\end{abstract}

Keywords: Rheumatoid arthritis, Rheumatoid arthritis fibroblast-like synoviocytes, Circ-AFF2, MiR-650, CNP

\footnotetext{
* Correspondence: Hghslh@163.com

${ }^{3}$ Department of Orthopedics, Peking University Medical Zibo Hospital, No.2,

5Th Street, Shanlv Xishan, Nanding Town, Zhangdian District, Zibo,

Shandong, China

Full list of author information is available at the end of the article
}

(c) The Author(s). 2021 Open Access This article is licensed under a Creative Commons Attribution 4.0 International License, which permits use, sharing, adaptation, distribution and reproduction in any medium or format, as long as you give appropriate credit to the original author(s) and the source, provide a link to the Creative Commons licence, and indicate if changes were made. The images or other third party material in this article are included in the article's Creative Commons licence, unless indicated otherwise in a credit line to the material. If material is not included in the article's Creative Commons licence and your intended use is not permitted by statutory regulation or exceeds the permitted use, you will need to obtain permission directly from the copyright holder. To view a copy of this licence, visit http://creativecommons.org/licenses/by/4.0/ The Creative Commons Public Domain Dedication waiver (http://creativecommons.org/publicdomain/zero/1.0/) applies to the data made available in this article, unless otherwise stated in a credit line to the data. 


\section{Introduction}

Rheumatoid arthritis (RA) is an autoimmune disease with common symptoms like musculoskeletal pain, swelling, and stiffness, which can impair physical function and life quality of patients [1]. The synovial tissue is a membranous organ lining joint cavities, which functions in joint destruction in RA [2]. Fibroblast-like synoviocytes are the dominant cells of synovial tissues, which contribute to cartilage destruction and RA development by interacting with immune cells and taking part in the inflammatory response of synovial joints [2, 3]. Moreover, fibroblast-like synoviocytes have important roles in the onset of RA [4]. Identification of fibroblast-like synoviocyte function in the present study fits into the framework of translational orthopedics by filling the gap between basic sciences and clinical sciences $[5,6]$. Therefore, illustrating the mechanism of fibroblast-like synoviocyte processes might help to understand RA pathogenesis.

Noncoding RNAs are involved in the autoimmunity and inflammation that play important roles in RA development [7]. Circular RNAs (circRNAs) are a type of closed noncoding RNAs which bind with microRNA (miRNA) to reduce miRNA activity, subsequently increasing mRNA stability, which have key roles in agingrelated diseases, including RA [8]. Moreover, the dysregulated circRNAs are associated with the function of fibroblast-like synoviocytes in RA [9]. For example, hsa circ_0088036 increases fibroblast-like synoviocyte proliferation and migration by modulating miR-140-3p and Sirtuin (SIRT)-1 [10]. CircRNA fragile mental retardation 2 (circ-AFF2; hsa_circ_0001947) is an upregulated circRNA in the peripheral blood of RA patients [11]. However, the function of this circRNA and how it participates in fibroblast-like synoviocyte processes in RA are mostly uncertain.

MiRNAs are another type of noncoding RNAs that have important functions in musculoskeletal conditions, such as RA, osteoarthritis, and tendon injuries [12-14]. Furthermore, miRNAs are related to the function of fibroblast-like synoviocytes in RA [15]. A previous study suggests miR-650 represses proliferation, migration, and invasion of rheumatoid arthritis synovial fibroblasts [16]. However, whether miR-650 is relevant to the function of circ-AFF2 in fibroblast-like synoviocytes remains uncertain. 2',3'-Cyclic nucleotide 3'phosphodiesterase (CNP) can prevent cartilage damage in inflammatory arthritis [17]. Moreover, CNP level is enhanced in RA patients [18]. Yet no study has been done to analyze the function of CNP in fibroblast-like synoviocytes.

In this study, we analyzed the function of circ-AFF2 on fibroblast-like synoviocyte processes in RA and explored the network of circ-AFF2/miR-650/CNP. This study might provide a novel insight into the pathology of RA.

\section{Materials and methods}

\section{Bioinformatic analysis}

The circular structure of circ-AFF2 (hsa_circ_0001947) was analyzed using CircView (http://gb.whu.edu.cn/ CircView/) [19]. The targets of circ-AFF2 were searched by starBase (http://starbase.sysu.edu.cn/) [20]. The targets of miR-650 were analyzed utilizing miRDB (http:// mirdb.org/) [21], miRwalk (http://mirwalk.umm.uniheidelberg.de/) [22], starBase, and Tarbase (http:// carolina.imis.athena-innovation.gr/diana_tools/web/ index.php? $r=$ tarbasev8/index) [23].

\section{Patients and tissues}

The synovial tissues of RA were obtained from RA patients $(n=34)$ who were diagnosed as RA and suffered from joint surgery at Weihai Municipal Hospital. All RA patients fulfilled the American College of Rheumatology criteria. The age- and gender-matched patients with joint trauma were regarded as the normal group, and the synovial tissues from the normal group $(n=23)$ were collected during the joint surgery. The patients with autoimmune or infectious disease were excluded from the normal group. All participants signed the written informed consent. This study complied with the guidelines of the Declaration of Helsinki and was authorized by the ethics committee of Weihai Municipal Hospital.

\section{RAFLS isolation and culture}

The fibroblast-like synoviocytes were isolated from synovial tissues of RA (RAFLSs) or normal subjects (normal cells). Briefly, the synovial tissues were cut into pieces and subjected to $0.1 \%$ type-I collagenase (Solarbio, Beijing, China) digestion at $37^{\circ} \mathrm{C}$ for $4 \mathrm{~h}$. After that, cells were collected after centrifugation at $1000 \mathrm{~g}$ for $5 \mathrm{~min}$ and cultured in Dulbecco's modified Eagle's medium (DMEM) (Gibco, Grand Island, NY, USA) plus 10\% fetal bovine serum (FBS) (Gibco) and 1\% penicillin/streptomycin (Gibco) at $37{ }^{\circ} \mathrm{C}$ with $5 \% \mathrm{CO}_{2}$.

\section{Quantitative reverse transcription polymerase chain reaction (qRT-PCR)}

RNA was extracted using Trizol (Vazyme, Nanjing, China) according to the instruction. The RNA from the nucleus or cytoplasm of RAFLSs was extracted using a cytoplasmic and nuclear RNA purification kit (Norgen Biotek, Thorold, Canada) according to the manufacturer's instruction. RNA (800 ng) was applied for cDNA synthesis using a miRNA or M-MLV reverse transcriptase kit or (Thermo Fisher Scientific, Waltham, MA, USA). The cDNA was mixed with SYBR (Vazyme) and primer pairs (Sangon, Shanghai, China) and utilized for qRT-PCR. The specific primer sequences are shown in Table 1. Relative RNA expression was calculated using 
Table 1 The primer sequences for qRT-PCR in this study

\begin{tabular}{|c|c|c|}
\hline \multirow[t]{2}{*}{ Name } & \multicolumn{2}{|l|}{ Sequence $\left(5^{\prime}-3^{\prime}\right)$} \\
\hline & Forward & Reverse \\
\hline miR-3612 & GCCGAGAGGAGGCATCTTGAG & AGTGCAGGGTCCGAGGTATT \\
\hline miR-650 & GCCGAGAGGAGGCAGCGCTC & AGTGCAGGGTCCGAGGTATT \\
\hline U6 & CAGGTCTCGGGAGAGAGATCG & TGTCGTCTTGGAGATCGGGAG \\
\hline circ-AFF2 & TCTTGGATGGAAAACCCAGT & AGTTTCCAAGCGTGTTCTGG \\
\hline CNP & GGAAAGCGCACGCTTAGGAG & GGCAGGAATGTGTGGCTIT \\
\hline ANKRD52 & TGGCGAGACCGGAATCCT & CCTIITCCAGGAGGGGTGG \\
\hline$A X L$ & CACGCGTAAACAACACGCA & GTTATGGGCTTCGCAGGAGA \\
\hline HNRNPUL1 & CTGCTITCTGGAGCCGAAGA & GGGTGCTCCTTGCTTCATCT \\
\hline APBB2 & CTCCTTTGTTTGCAGGGATTTTGC & TGGCATTCTTCCGTTCAGCC \\
\hline RAC1 & TGATGCAGGCCATCAAGTGT & AGAACACATCTGTTTGCGGA \\
\hline CTSB & ATCATGTGGGTGAGCCAGTG & TGGGGCAGCGAGAAGTTAAG \\
\hline PLEC & TCATCCAGGCCTACGAGGAG & CCAGCAGGGAGATGAGGTTG \\
\hline CD274 & TTGCTGAACGCCCCATACAA & CCCCGATGAACCCCTAAACC \\
\hline GAPDH & TTCTITGCGTCGCCAGGTG & GGAGGGAGAGAACAGTGAGC \\
\hline
\end{tabular}

the $2^{-\Delta \Delta C t}$ method, with U6 or GAPDH as an internal control.

\section{Cell transfection}

Circ-AFF2 overexpression vector was constructed by Geneseed (Guangzhou, China), with pCD5-ciR vector as a negative control (vector). CNP overexpression vector was constructed in our laboratory, with pcDNA3.1 (Thermo Fisher Scientific, Waltham, MA, USA) as a negative control (pcDNA). Small interfering RNA (siRNA) for circ-AFF2 (si-circ-AFF2), siRNA negative control (si-NC), miR-3612 mimic, miR-650 mimic, mimic negative control (miR-NC), miR-650 inhibitor (anti-miR-650), and inhibitor negative control (anti-NC) were generated by Genomeditech (Shanghai, China), and the related sequences are displayed in Table 2. RAFLSs were transfected with $2 \mu \mathrm{g}$ vectors or $40 \mathrm{nM}$ oligonucleotides using Lipofectamine 3000 (Thermo Fisher Scientific) for $24 \mathrm{~h}$ according to the instruction.

Table 2 The oligo sequences for transfection in this study

\begin{tabular}{ll}
\hline Name & Sequence (5'-3') \\
\hline si-Circ-AFF2 & ACCUUUGUUUGUUUCACUUGU \\
si-NC & UGGAAAGAAACAUUCACUUGU \\
miR-3612 mimic & AGGAGGCAUCUUGAGAAAUGGA \\
miR-650 mimic & AGGAGGCAGCGCUCUCAGGAC \\
\multicolumn{1}{c}{ miR-NC } & CGAUCGCAUCAGCAUCGAUUGC \\
anti-miR-650 & GUCCUGAGAGCGCUGCCUCCU \\
anti-NC & CAGUACUUUUGUGUAGUACAA \\
\hline
\end{tabular}

\section{Cell Counting Kit-8 (CCK-8)}

RAFLSs $\left(1 \times 10^{4} /\right.$ well $)$ were dispersed in 96-well plates. After $0,24,48$, or $72 \mathrm{~h}, 10 \mu \mathrm{L}$ CCK-8 (Solarbio) was infused, and cells were continuously nurtured for $3 \mathrm{~h}$. The optical density (OD) level at $450 \mathrm{~nm}$ was detected using a microplate reader (Bio-Rad, Hercules, CA, USA).

\section{Enzyme-linked immunosorbent assay (ELISA)}

The secretion levels of pro-inflammatory interleukin (IL)- 6 and anti-inflammatory IL-10 were detected by ELISA. In brief, $5 \times 10^{4}$ RAFLSs were placed in 24-well plates and incubated for $48 \mathrm{~h}$. The culture supernatants were collected and used for the detection of IL-6 and IL10 secretion levels using an IL-6 or IL-10 ELISA kit (Thermo Fisher Scientific) following the instructions. The absorbance at $450 \mathrm{~nm}$ was detected with a microplate reader.

\section{Flow cytometry}

An Annexin V-fluorescein isothiocyanate (FITC) apoptosis detection kit (Beyotime, Shanghai, China) was used for apoptosis detection according to the instruction. In brief, $2 \times 10^{5}$ RAFLSs were dispersed in 12-well plates for $72 \mathrm{~h}$. Then cells were collected, resuspended in Annexin V binding buffer, and stained with $10 \mu \mathrm{L}$ Annexin V-FITC and propidium iodide (PI) for $10 \mathrm{~min}$ in the dark. A flow cytometer (Beckman Coulter, Brea, CA, USA) was used to detect the apoptotic rate.

\section{Caspase 3 activity analysis}

Caspase 3 activity was analyzed using a caspase 3 activity assay kit (Beyotime) following the instructions. In brief, RAFLSs were incubated for $72 \mathrm{~h}$, and then $2 \times 10^{6}$ cells 
were collected and lysed in $100 \mu \mathrm{L}$ Caspase cell lysis buffer. Next, $50 \mu \mathrm{L}$ lysates were placed into 96-well plates and incubated with $40 \mu \mathrm{L}$ Caspase reaction buffer and $10 \mu \mathrm{L} 2 \mathrm{mM}$ Ac-DEVD-AFC at $37^{\circ} \mathrm{C}$ for $120 \mathrm{~min}$. The absorbance at $405 \mathrm{~nm}$ was examined through a microplate reader. Relative caspase 3 activity was shown as the fold change of the control group.

\section{Transwell analysis}

Transwell migration analysis was processed using 24well Transwell chambers (Corning Inc., Corning, NY, USA) with a fibronectin-coated polycarbonate membrane. $1 \times 10^{5}$ RAFLSs in serum-free DMEM were placed in the upper chambers, and $500 \mu \mathrm{L}$ DMEM with $10 \% \mathrm{FBS}$ was infused in the lower chambers. After $24 \mathrm{~h}$, RAFLSs that passed the membrane were stained with $0.1 \%$ crystal violet (Solarbio) and imaged under $\mathrm{a} \times 100$ magnification microscope (Olympus, Tokyo, Japan). For Transwell invasion analysis, the membranes were precoated by Matrigel (Solarbio), and $5 \times 10^{5}$ RAFLSs in non-serum DMEM were dispersed in the upper chambers. The other procedures were the same with the Transwell migration analysis. The migratory or invasive cell numbers were analyzed by Image J (NIH, Bethesda, MD, USA).

\section{Western blotting}

After isolating in radioimmunoprecipitation assay buffer (Solarbio), protein was collected by centrifugation at 10 , $000 \times g$ for $10 \mathrm{~min}$, and the concentration was detected using a bicinchoninic acid kit (Thermo Fisher Scientific). Twenty-microgram samples were run on sodium dodecyl sulfate-polyacrylamide gel electrophoresis and transferred to nitrocellulose membrane (Solarbio). The membranes were blocked in 3\% bovine serum albumin (Solarbio) for $1 \mathrm{~h}$ and then incubated with Abcam primary antibodies overnight and secondary antibody (Abcam, Cambridge, UK) for $2 \mathrm{~h}$. The antibodies included E-cadherin (ab133597, 1:1000 dilution), Ncadherin (ab18203, 1:2000 dilution), Vimentin (ab92547, 1:2000 dilution), CNP (ab183500, 1:1000 dilution), Rac family small GTPase 1 (RAC1) (ab155938, 1:500 dilution), GAPDH (ab37168, 1:3000 dilution), and horseradish peroxidase-labeled IgG (ab97051, 1:5000 dilution). After exposure to enhanced chemiluminescence (Solarbio), the blots were analyzed by Image J, with GAPDH as a normalized reference.

Dual-luciferase reporter, RNA immunoprecipitation (RIP), and pull-down assays

The wild-type (WT) luciferase reporter vectors (WTcirc-AFF2 and WT-CNP 3'UTR) were constructed by inserting the sequence of circ-AFF2 or CNP 3'UTR containing miR-650 or miR-3612 complementary sites in the pmir-GLO vector (Promega, Madison, WI, USA). The mutant (MUT) luciferase reporter vectors (MUTcirc-AFF2 and MUT-CNP 3'UTR) were constructed by mutating the binding sites of miR-650 or miR-3612. For the dual-luciferase reporter assay, these constructed vectors together with miR-3612 mimic, miR-650 mimic, or miR-NC were transfected in RAFLSs. Luciferase activity was measured with a dual-luciferase analysis kit (Promega) after $24 \mathrm{~h}$ post-transfection.

An EZ-Magna RIP kit (Sigma, St. Louis, MO, USA) was utilized for RIP assay. In brief, $1 \times 10^{7}$ RAFLSs were lysed in RIP lysis buffer and incubated with Anti-IgG or Anti-Ago2-coated magnetic beads for $6 \mathrm{~h}$. RNA on the beads was extracted, and enriched circ-AFF2, miR-3612, and miR-650 levels were detected by qRT-PCR as mentioned above.

RNA pull-down assay was performed using a Magnetic RNA Pull-Down Kit (Thermo Fisher Scientific). The biotinylated miR-3612 mimic (Bio-miR-3612), Bio-miR-650, and negative control (Bio-miR-NC) were generated by RiboBio (Guangzhou, China). RAFLSs were transfected with $100 \mathrm{~nm}$ biotinylated probes for $48 \mathrm{~h}$ and then were lysed using lysis buffer. The lysates were incubated with streptavidin-coated magnetic beads overnight. Then RNA enriched on the beads was isolated, and circ-AFF2 enrichment level was measured by qRT-PCR.

\section{Statistical analysis}

Three independent experiments were performed. Data were shown as mean \pm standard deviation (SD). The comparison between groups was conducted by the Student $t$-test or one-way analysis of variance followed via Tukey's post hoc test as appropriate, processed using GraphPad Prism 8 (GraphPad Inc., La Jolla, CA, USA). The difference was statistically significant at $P<0.05$.

\section{Results \\ Circ-AFF2 level is increased in RA}

Circ-AFF2 (hsa_circ_0001947) was studied by CircView, which showed that circ-AFF2 was back-spliced by the exon 3 of AFF2 transcripts (Fig. 1a). To explore whether circ-AFF2 was related to RA development, circ-AFF2 expression change was analyzed in synovial tissues from RA $(n=34)$ or normal subjects $(n=23)$. Compared with normal subjects, circ-AFF2 level was markedly enhanced in RA synovial samples (Fig. 1b). Furthermore, the fibroblast-like synoviocytes were isolated from RA tissues (RAFLSs) or normal subjects (normal cells). CircAFF2 expression was higher in RAFLSs than in normal cells (Fig. 1c). Additionally, circ-AFF2 expression distribution was analyzed by using GAPDH and U6 as a cytoplasm or nucleus reference. Results showed circ-AFF2 was mostly located in the cytoplasm of RAFLSs (Fig. 1d). 


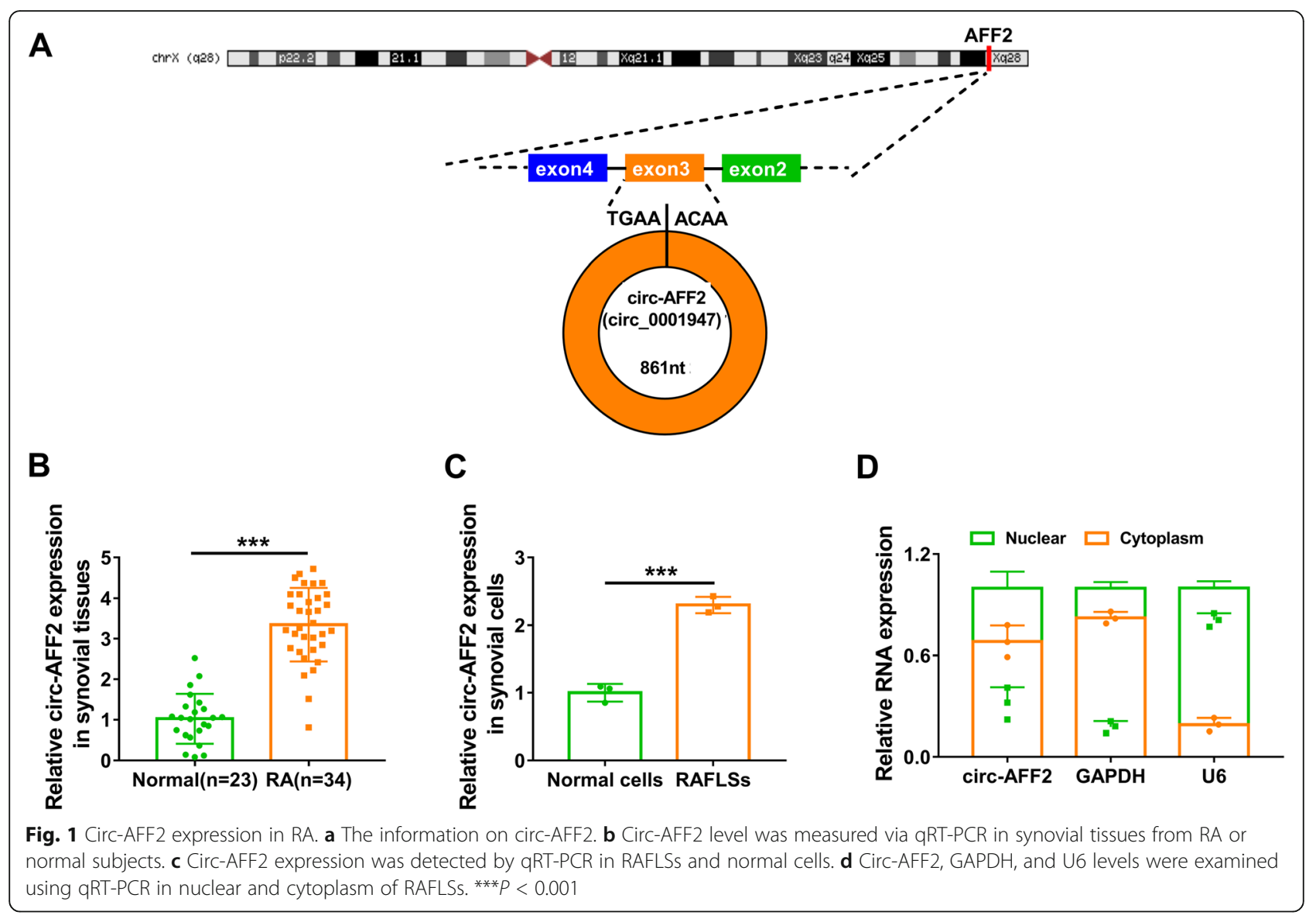

These results suggested circ-AFF2 might have important roles in RA development.

\section{Circ-AFF2 promotes cell proliferation, inflammatory response, migration, and invasion}

To study the role of circ-AFF2 in RAFLS processes, circAFF2 was overexpressed or knocked down in RAFLSs. Circ-AFF2 expression level in RAFLSs was increased 11.94-fold by transfection of circ-AFF2 overexpression vector, and decreased $80 \%$ by addition of si-circ-AFF2 (Fig. 2a). RAFLS proliferation was significantly promoted by circ-AFF2 overexpression, but decreased by circ-AFF2 knockdown (Fig. 2b). Moreover, circ-AFF2 overexpression evidently increased secretion of IL-6 and decreased IL-10 level in RAFLSs, while silencing circ-AFF2 led to an opposite effect (Fig. 2c, d). Additionally, circ-AFF2 upregulation clearly reduced cell apoptosis and caspase 3 activity in RAFLSs, but circ-AFF2 knockdown caused an opposite effect (Fig. 2e, f). Furthermore, circ-AFF2 addition markedly enhanced migration and invasion of RAFLSs, but silencing circ-AFF2 inhibited the migratory and invasive abilities (Fig. 2g). In addition, epithelial-mesenchymal transition (EMT) was associated with cell migration and invasion, and related protein level was detected in RAFLSs. Results showed that circ-AFF2 overexpression notably resulted in reduction of E-cadherin and elevation of $\mathrm{N}$-cadherin and Vimentin, while circ-AFF2 knockdown played opposite effects on these protein levels (Fig. 2h). These data indicated circ-AFF2 might promote RAFLS proliferation, inflammatory response, migration, and invasion.

\section{MiR-650 is targeted by circ-AFF2}

To explore the regulatory network addressed by circAFF2, we predicted the targets of circ-AFF2 (hsa_circ 0001947) by starBase. Two miRNAs (miR-3612 and miR-650) were predicted as the potential targets of circAFF2 (Fig. 3a). Predicted binding sequences are displayed in Fig. 3b, and WT-circ-AFF2 and MUT-circAFF2 luciferase reporter vectors were constructed. The luciferase activity of WT-circ-AFF2 was obviously decreased by miR-650 mimic, but it was not affected by miR-3612 mimic (Fig. 3c). Moreover, the effect of miR650 mimic on the luciferase activity was abolished in the MUT-circ-AFF2 group (Fig. 3c). In addition, the RIP assay showed circ-AFF2, miR-3612, and miR-650 might be enriched in an Ago2-dependent manner (Fig. 3d). And RNA pull-down analysis displayed circ-AFF2 could bind with miR-650, but not with miR-3612 (Fig. 3e). Furthermore, the overexpression or knockdown efficacy of miR-650 mimic or inhibitor (anti-miR-650) is 


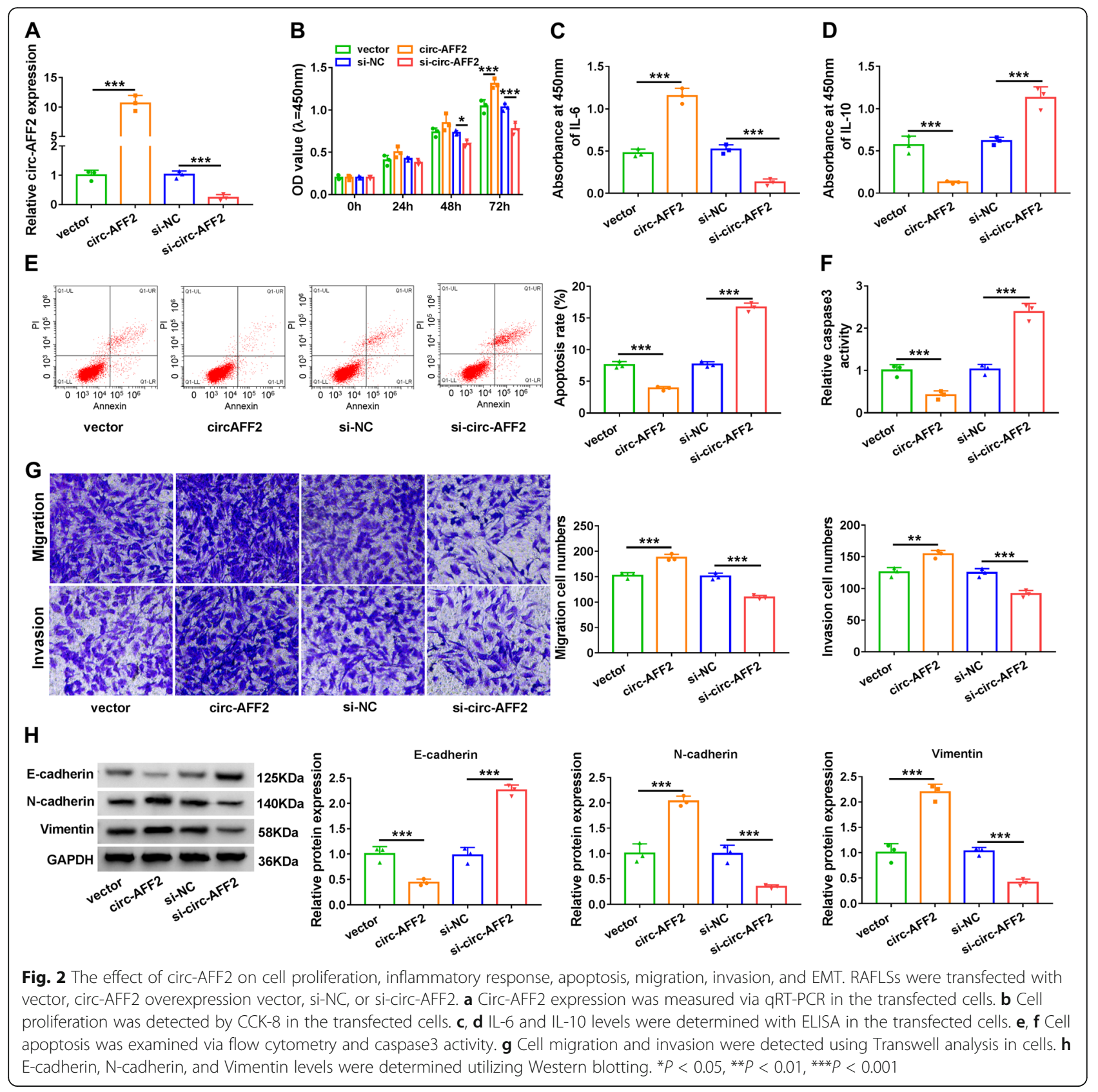

confirmed in Fig. 3f. Additionally, the influence of circAFF2 on miR-650 expression was investigated in RAFL Ss. Results showed circ-AFF2 overexpression evidently reduced miR-650 level, while circ-AFF2 knockdown enhanced miR-650 expression (Fig. 3g). These results suggested circ-AFF2 could target miR-650.

\section{MiR-650 knockdown attenuates the effect of silencing} circ-AFF2 on RAFLS proliferation, inflammatory response, migration, and invasion

To probe if miR-650 was related to circ-AFF2-mediated cell processes, RAFLSs were transfected with si-NC, si-
circ-AFF2, and si-circ-AFF2 + anti-NC or anti-miR-650. The transfection of anti-miR-650 weakened silencing circ-AFF2-induced miR-650 upregulation (Fig. 4a). MiR650 knockdown rescued interference of circ-AFF2mediated proliferation reduction (Fig. 4b). In addition, miR-650 downregulation reversed knockdown of circAFF2-mediated inhibition of IL- 6 secretion and increase of IL-10 (Fig. 4c, d). Furthermore, miR-650 knockdown weakened interference of circ-AFF2-induced promotion of apoptosis and caspase 3 activity (Fig. 4e, f). Additionally, miR-650 downregulation attenuated knockdown of circ-AFF2-mediated inhibition of cell migration and 


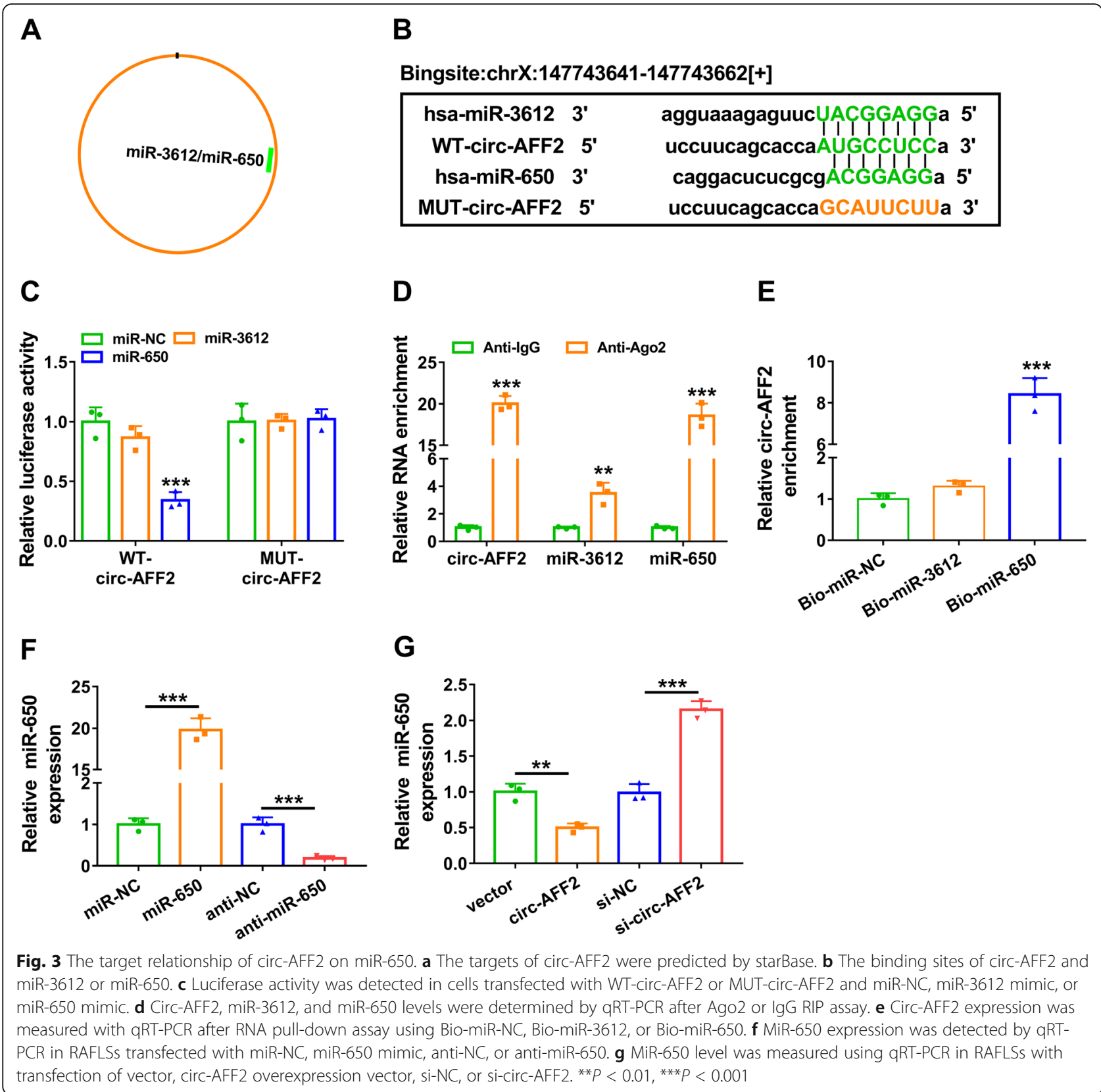

invasion (Fig. 4g). Moreover, miR-650 knockdown reversed the elevation of E-cadherin and the decrease of $\mathrm{N}$-cadherin and Vimentin mediated by silencing circAFF2 (Fig. 4h). These results indicated circ-AFF2 modulated RAFLS processes by regulating miR- 650 .

\section{CNP is targeted by miR-650 and regulated via the circ- AFF2/miR-650 axis}

To further explore the regulatory network, the targets of miR-650 were analyzed by miRDB, miRwalk, starBase, and Tarbase. There were 9 overlapping targets predicted by the four tools online (Fig. 5a). The effect of miR-650 on their expression levels was investigated in RAFLSs. As shown in
Fig. 5b, c, CNP level was reduced most by miR-650 overexpression. Hence, CNP was selected as a potential target for further experiments. Predicted binding sites between miR650 and CNP are displayed in Fig. 5d. To validate this prediction, WT-CNP 3'UTR and MUT-CNP 3'UTR luciferase reporter vectors were constructed. The luciferase activity of WT-CNP 3'UTR was clearly reduced by miR-650 mimic, while the activity of MUT-CNP 3'UTR was not affected via miR-650 (Fig. 5e). Moreover, CNP expression was negatively regulated by miR-650 (Fig. 5f, g). In addition, circAFF2 overexpression or downregulation obviously increased or decreased CNP expression in RAFLSs, and this effect was reversed by miR-650 mimic or anti-miR-650 


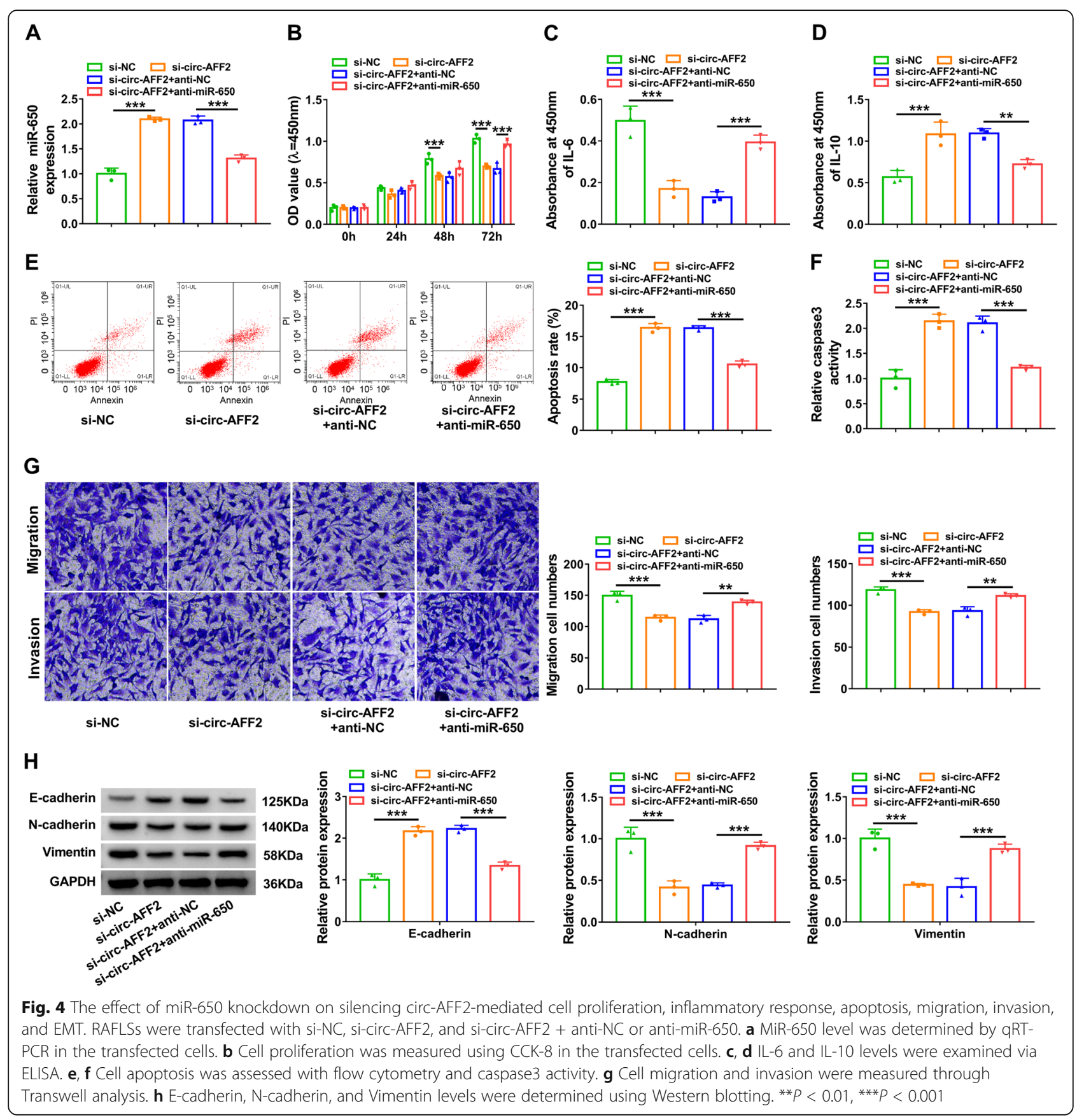

(Fig. 5h, i). These data suggested circ-AFF2 could target CNP through miR-650.

\section{MiR-650 constrains cell proliferation, inflammatory response, migration, and invasion by decreasing CNP} To explore the function of the miR-650/CNP axis in RAFL $\mathrm{S}$ processes, cells were transfected with miR-NC, miR-650 mimic, and miR-650 mimic + pcDNA or CNP overexpression vector. The transfection of CNP overexpression vector effectively enhanced CNP expression in RAFLSs (Fig. 6a, b). Moreover, addition of CNP overexpression vector restored CNP level that was decreased by miR-650 mimic (Fig. 6c, d). CCK-8 assay showed miR-650 mimic evidently reduced RAFLS proliferation, and CNP upregulation reversed this effect (Fig. 6e). Additionally, miR-650 overexpression significantly constrained IL-6 expression and increased IL-10 secretion in RAFLSs, and these events were reversed by CNP overexpression (Fig. 6f, g). Furthermore, miR-650 overexpression clearly caused apoptosis promotion and increased caspase 3 activity, and this effect was weakened via CNP upregulation (Fig. 6h, i). In addition, miR-650 markedly restrained cell migration, invasion, and 

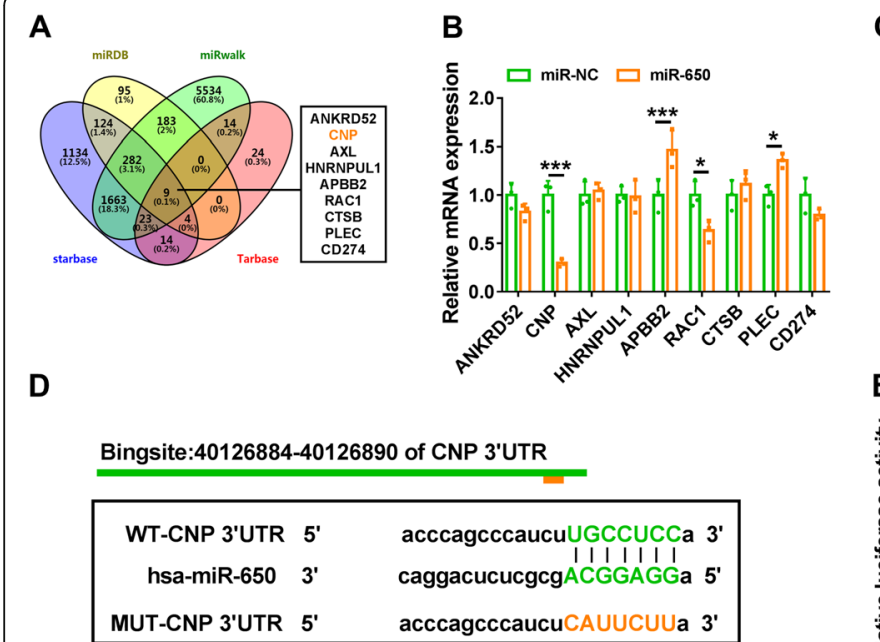

G
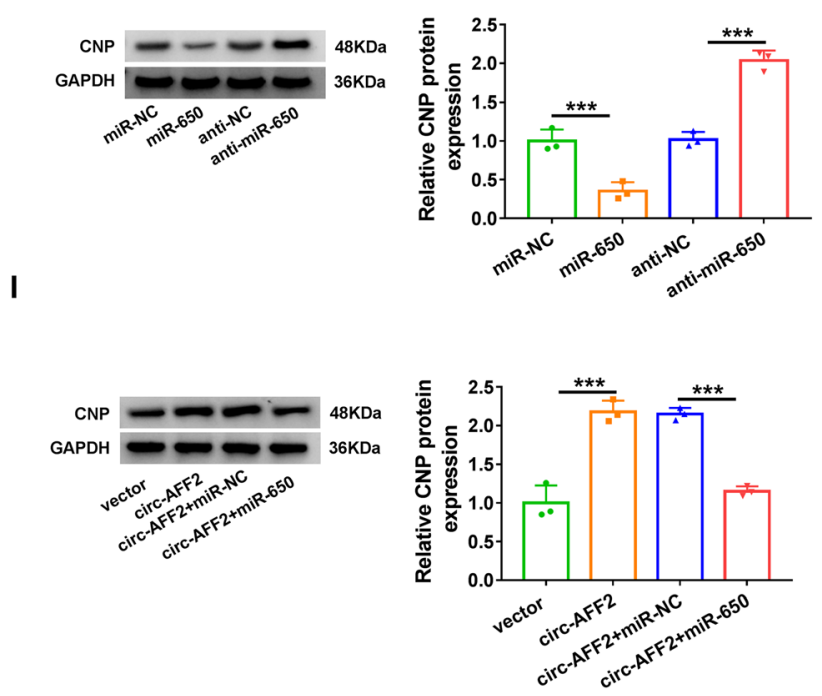

C
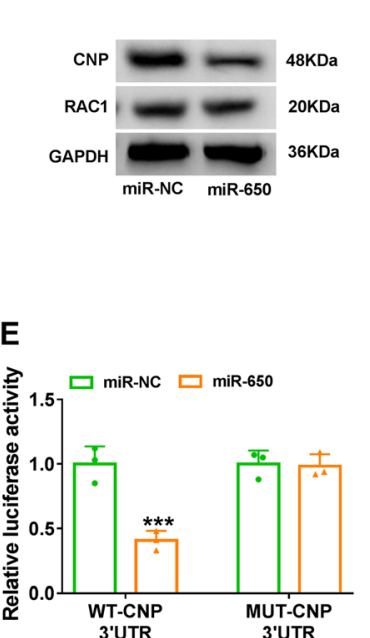

H
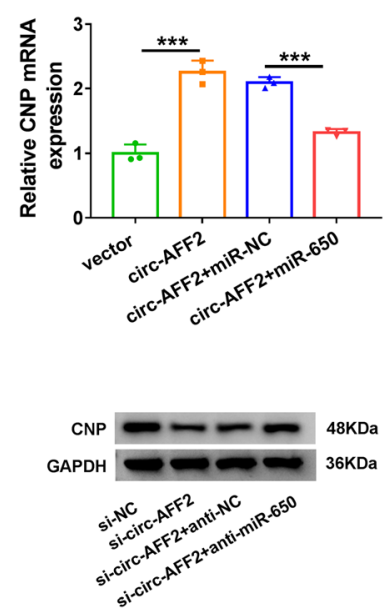
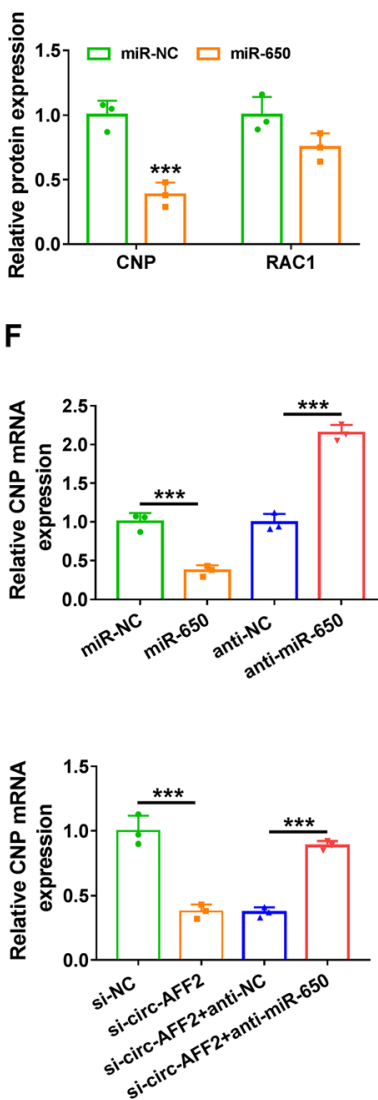

$\mathbf{F}$
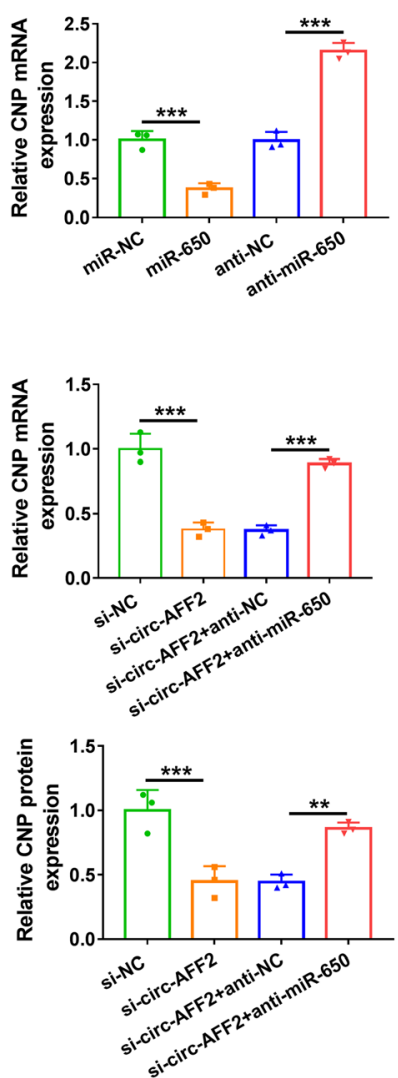

Fig. 5 The target association of miR-650 and CNP. a The targets of miR-650 were predicted by miRDB, miRwalk, starBase, and Tarbase. b The levels of the predicted targets were detected by qRT-PCR in cells transfected with miR-NC or miR-650 mimic. c CNP and RAC1 protein levels were detected via Western blotting in cells transfected with miR-NC or miR-650 mimic. $\mathbf{d}$ The target sites of miR-650 and CNP. e Luciferase activity was measured in cells transfected with WT-CNP 3'UTR or MUT-CNP 3'UTR and miR-NC or miR-650 mimic. f, $\mathbf{g}$ CNP level was measured via qRT-PCR and Western blotting in RAFLSs transfected with miR-NC, miR-650 mimic, anti-NC, or anti-miR-650. h, i CNP level was determined by qRT-PCR and Western blotting in RAFLSs with transfection of vector, circ-AFF2 overexpression vector, circ-AFF2 overexpression vector + miR-NC or miR-650 mimic, si-NC, si-circ-AFF2, and si-circ-AFF2 + anti-NC or anti-miR-650. ${ }^{*} P<0.05,{ }^{* *} P<0.01,{ }^{* * *} P<0.001$

EMT, which was mitigated by CNP addition (Fig. 6j-1). These results indicated miR-650 inhibited RAFLS processes by regulating CNP.

\section{Discussion}

RA is a joint disease characterized by the chronic inflammation of the synovial membrane, and significant improvement is involved in the diagnosis and therapy of RA [24]. However, approximately 20-25\% of patients do not reach the low disease activity [24].
Fibroblast-like synoviocytes are involved in the overproduction of enzymes and cytokines that are associated with cartilage degradation and immune cell infiltration [25]. CircRNAs are involved in various diseases including RA, but their roles have not been broadly explored [26]. Here we explored the role of circ-AFF2 in RA and found the promoting function of circ-AFF2 on RAFLS processes. Moreover, we confirmed it was associated with the miR-650/CNP axis. 


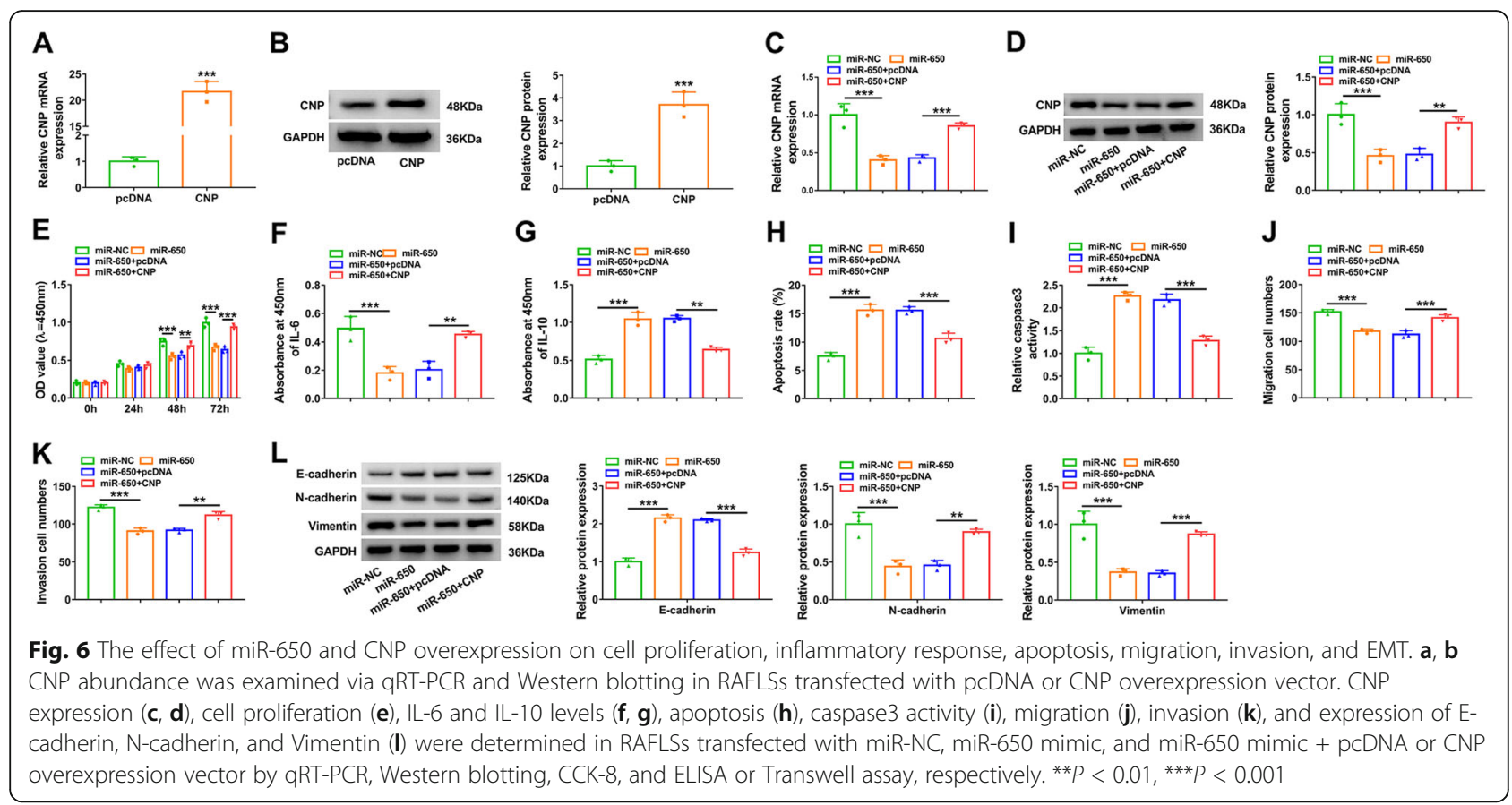

Our study showed upregulated circ-AFF2 in RA synovial tissues and RAFLSs, which was also consistent with that in the peripheral blood of RA patients [11]. This finding suggested RA development and RAFLS processes might be related to the dysregulated circ-AFF2. Targeting RAFLS apoptosis is an important strategy for RA treatment [27]. Here we found silencing circ-AFF2 attenuated cell proliferation and promoted apoptosis. Furthermore, the balance of pro-inflammatory IL- 6 and anti-inflammatory IL-10 regulates the inflammatory response in RA [28-30]. By detecting their secretion levels, we confirmed circ-AFF2 knockdown mitigated the inflammatory response in RAFLSs by decreasing IL-6 and increasing IL-10. Additionally, the migration, invasion, and gain of mesenchymal characteristics of RAFLSs are also key events in RA [31-33]. By measuring cell migration, invasion, and EMT-related protein expression [34], we found that circ-AFF2 knockdown repressed RAFLS migration and invasion. Moreover, circ-AFF2 overexpression promoted RAFLS proliferation, inflammatory response, migration, and invasion. Collectively, circ-AFF2 might serve as an important intervention for RA therapy.

Moreover, the regulatory network mediated by circAFF2 was explored. We firstly confirmed circ-AFF2 could sponge miR-650. Previous studies reported miR650 promoted cell proliferation, migration, and invasion in oral cancer and hepatocellular carcinoma [35, 36], while miR-650 suppressed cell proliferation in glioma and acute myeloid leukemia [37, 38]. More importantly, a former work indicated miR-650 suppressed proliferation, migration, and invasion of RAFLSs by modulating protein kinase B2 (AKT2) [16]. Similarly, we also found this function of miR-650 in our study. Moreover, we confirmed miR-650 was modulated by circ-AFF2 to take part in the regulation of RAFLS processes. Additionally, we further confirmed CNP was a downstream target of miR-650. Multiple evidences suggested CNP had a proproliferation role in various cell lines, like melanoma cells, mouse Leydig cells, and osteoblastic cells [39-41]. However, the function of CNP on RAFLS processes remains unclear. Our study found CNP upregulation attenuated the suppressive role of miR-650 in RAFLS proliferation, migration, and invasion. In addition, we firstly confirmed circ-AFF2 regulated CNP expression indirectly through miR-650. In this way, this axis promoted RAFLS proliferation, inflammatory response, migration, and invasion, thereby contributing to RA development.

Our study performed three independent experiments using the primary RAFLSs, which might represent the physiology of RA patients. Moreover, we firstly confirmed the involvement of the circ-AFF2/miR-650/CNP network in RAFLS dysfunction, indicating the importance and clinical expectation of this axis in RA progression and treatment. However, there were some limitations in the current study. First, we measured circAFF2 expression in RA patients $(n=34)$, and increased sample sizes are needed in a further study. Second, the downstream pathway mediated by the circ-AFF $2 / \mathrm{miR}$ 650/CNP network should be explored. Third, our conclusions were based on the in vitro experiments, and animal studies are needed in the future. 


\section{Conclusion}

Circ-AFF2 upregulation facilitated proliferation, inflammatory response, migration, and invasion of RAFLSs, partly by the miR-650/CNP axis. This study provided a new regulatory network of circ-AFF2/miR-650/CNP in RAFLSs. We expect this work will serve as a valuable resource in the future clinical treatment of RA.

\section{Abbreviations}

RA: Rheumatoid arthritis; RAFLSs: Rheumatoid arthritis fibroblast-like synoviocytes; CNP: Cyclic nucleotide 3'-phosphodiesterase; EMT: Epithelialmesenchymal transition; ELISA: Enzyme-linked immunosorbent assay; FITC: Fluorescein isothiocyanate

\section{Acknowledgements}

Not applicable

\section{Authors' contributions}

WQ designed and performed the research. $\amalg$ and $\mathrm{GH}$ analyzed the data. WQ wrote the manuscript. The authors read and approved the final manuscript.

\section{Funding}

Not applicable

\section{Availability of data and materials}

Not applicable

\section{Ethics approval and consent to participate}

Written informed consent was obtained from all participants, and this study was permitted by the Ethics Committee of Weihai Municipal Hospital.

\section{Consent for publication}

Not applicable

\section{Competing interests}

The authors declare that they have no competing interests.

\section{Author details}

'Department of Joint and Sports Medicine, Weihai Municipal Hospital, Cheeloo College of Medicine, Shandong University, Weihai 264200, Shandong, China. ${ }^{2}$ Department of Medical, Zibo Social Welfare Institute, Zibo, Shandong, China. ${ }^{3}$ Department of Orthopedics, Peking University Medical Zibo Hospital, No.2, 5Th Street, Shanlv Xishan, Nanding Town, Zhangdian District, Zibo, Shandong, China.

Received: 7 December 2020 Accepted: 17 February 2021

Published online: 02 March 2021

\section{References}

1. Sparks JA. Rheumatoid arthritis. Ann Intern Med. 2019;170:ITC1-ITC16.

2. Yoshitomi H. Regulation of immune responses and chronic inflammation by fibroblast-like synoviocytes. Front Immunol. 2019;10:1395.

3. Masoumi M, Bashiri H, Khorramdelazad H, Barzaman K, Hashemi N, Sereshki $\mathrm{HA}$, et al. Destructive roles of fibroblast-like synoviocytes in chronic inflammation and joint damage in rheumatoid arthritis. Inflammation. 2020.

4. Karami J, Aslani S, Tahmasebi MN, Mousavi MJ, Sharafat Vaziri A, Jamshidi A, et al. Epigenetics in rheumatoid arthritis; fibroblast-like synoviocytes as an emerging paradigm in the pathogenesis of the disease. Immunol Cell Biol. 2020:98:171-86.

5. Mediouni M, Schlatterer DR, Madry H, Cucchiarini M, Rai B. A review of translational medicine. The future paradigm: how can we connect the orthopedic dots better? Curr Med Res Opin. 2018:34:1217-29.

6. Worboys M, Toon E. Special issue-before translational medicine: laboratory clinic relations lost in translation? Cortisone and the treatment of rheumatoid arthritis in Britain, 1950-1960. Hist Philos Life Sci. 2019;41:54.

7. Wang J, Yan S, Yang J, Lu H, Xu D, Wang Z. Non-coding RNAs in rheumatoid arthritis: from bench to bedside. Front Immunol. 2019;10:3129.
8. $\quad$ Ren S, Lin P, Wang J, Yu H, Lv T, Sun L, et al. Circular RNAs: promising molecular biomarkers of human aging-related diseases via functioning as an miRNA sponge. Mol Ther Methods Clin Dev. 2020;18:215-29.

9. Taheri M, Eghtedarian R, Dinger ME, Ghafouri-Fard S. Dysregulation of noncoding RNAs in rheumatoid arthritis. Biomed Pharmacother. 2020;130: 110617

10. Zhong S, Ouyang Q, Zhu D, Huang Q, Zhao J, Fan M, et al. Hsa_circ_ 0088036 promotes the proliferation and migration of fibroblast-like synoviocytes by sponging miR-140-3p and upregulating SIRT 1 expression in rheumatoid arthritis. Mol Immunol. 2020;125:131-9.

11. Luo Q, Liu J, Fu B, Zhang L, Guo Y, Huang Z, et al. Circular RNAs Hsa_circ 0002715 and Hsa_circ_0035197 in peripheral blood are novel potential biomarkers for new-onset rheumatoid arthritis. Dis Markers. 2019;2019: 2073139.

12. Najm A, Blanchard F, Le Goff B. Micro-RNAs in inflammatory arthritis: from physiopathology to diagnosis, prognosis and therapeutic opportunities. Biochem Pharmacol. 2019:165:134-44.

13. Oliviero A, Della Porta G, Peretti GM, Maffulli N. MicroRNA in osteoarthritis: physiopathology, diagnosis and therapeutic challenge. Br Med Bull. 2019. 130:137-47.

14. Giordano L, Porta GD, Peretti GM, Maffulli N. Therapeutic potential of microRNA in tendon injuries. Br Med Bull. 2020;133:79-94.

15. Iwamoto N, Kawakami A. Recent findings regarding the effects of microRNAs on fibroblast-like synovial cells in rheumatoid arthritis. Immunol Med. 2019;42:156-61.

16. Xu X, Chen H, Zhang Q, Xu J, Shi Q, Wang M. MiR-650 inhibits proliferation, migration and invasion of rheumatoid arthritis synovial fibroblasts by targeting AKT2. Biomed Pharmacother. 2017;88:535-41.

17. Bukulmez H, Khan F, Bartels CF, Murakami S, Ortiz-Lopez A, Sattar A, et al. Protective effects of C-type natriuretic peptide on linear growth and articular cartilage integrity in a mouse model of inflammatory arthritis. Arthritis Rheumatol. 2014:66:78-89.

18. Olewicz-Gawlik A, Trzybulska D, Grala P, Hrycaj P. Blood serum levels of amino-terminal pro-C-type natriuretic peptide in patients with rheumatoid arthritis. Adv Med Sci. 2010;55:261-5.

19. Feng J, Xiang Y, Xia S, Liu H, Wang J, Ozguc FM, et al. CircView: a visualization and exploration tool for circular RNAs. Brief Bioinform. 2018;19: 1310-6.

20. Li JH, Liu S, Zhou H, Qu LH, Yang JH. starBase v2.0: decoding miRNA-ceRNA, miRNA-ncRNA and protein-RNA interaction networks from large-scale CLIPSeq data. Nucleic Acids Res. 2014;42:D92-7.

21. Chen $Y$, Wang $X$. miRDB: an online database for prediction of functional microRNA targets. Nucleic Acids Res. 2020;48:D127-D31.

22. Sticht C, De La Torre C. Parveen A. Gretz N. miRWalk: an online resource for prediction of microRNA binding sites. PLoS One. 2018;13:e0206239.

23. Karagkouni D, Paraskevopoulou MD, Chatzopoulos S, Vlachos IS, Tastsoglou S, Kanellos I, et al. DIANA-TarBase v8: a decade-long collection of experimentally supported miRNA-gene interactions. Nucleic Acids Res. 2018;46:D239-D45.

24. Aletaha D, Smolen JS. Diagnosis and management of rheumatoid arthritis: a review. JAMA. 2018;320:1360-72.

25. Falconer J, Murphy AN, Young SP, Clark AR, Tiziani S, Guma M, et al. Review: Synovial cell metabolism and chronic inflammation in rheumatoid arthritis. Arthritis Rheumatol. 2018:70:984-99.

26. Lodde V, Murgia G, Simula ER, Steri M, Floris M, Idda ML. Long noncoding RNAs and circular RNAs in autoimmune diseases. Biomolecules. 2020;10.

27. Zhang Q, Liu J, Zhang M, Wei S, Li R, Gao Y, et al. Apoptosis induction of fibroblast-like synoviocytes is an important molecular-mechanism for herbal medicine along with its active components in treating rheumatoid arthritis. Biomolecules. 2019;9.

28. Garbers C, Heink S, Korn T, Rose-John S. Interleukin-6: designing specific therapeutics for a complex cytokine. Nat Rev Drug Discov. 2018;17:395-412.

29. Saxena A, Khosraviani S, Noel S, Mohan D, Donner T, Hamad AR. Interleukin10 paradox: a potent immunoregulatory cytokine that has been difficult to harness for immunotherapy. Cytokine. 2015:74:27-34.

30. Ireland SJ, Monson NL, Davis LS. Seeking balance: potentiation and inhibition of multiple sclerosis autoimmune responses by IL-6 and IL-10. Cytokine. 2015;73:236-44.

31. Steenvoorden MM, Tolboom TC, van der Pluijm G, Lowik C, Visser CP, DeGroot J, et al. Transition of healthy to diseased synovial tissue in rheumatoid arthritis is associated with gain of mesenchymal/fibrotic characteristics. Arthritis Res Ther. 2006:8:R165. 
32. Li G, Zhang Y, Qian Y, Zhang H, Guo S, Sunagawa M, et al. Interleukin-17A promotes rheumatoid arthritis synoviocytes migration and invasion under hypoxia by increasing MMP2 and MMP9 expression through NF-kappaB/ HIF-1alpha pathway. Mol Immunol. 2013;53:227-36.

33. Zhu D, Zhao J, Lou A, Huang Q, OuYang Q, Zhu J, et al. Transforming growth factor beta1 promotes fibroblast-like synoviocytes migration and invasion via TGF-beta1/Smad signaling in rheumatoid arthritis. Mol Cell Biochem. 2019:459:141-50.

34. Serrano-Gomez SJ, Maziveyi M, Alahari SK. Regulation of epithelialmesenchymal transition through epigenetic and post-translational modifications. Mol Cancer. 2016;15:18.

35. Ningning S, Libo S, Chuanbin W, Haijiang S, Qing Z. MiR-650 regulates the proliferation, migration and invasion of human oral cancer by targeting growth factor independent 1 (Gfi1). Biochimie. 2019;156:69-78.

36. Qin A, Wu J, Zhai M, Lu Y, Huang B, Lu X, et al. Axin1 inhibits proliferation, invasion, migration and EMT of hepatocellular carcinoma by targeting miR650. Am J Transl Res. 2020;12:1114-22.

37. Xu L, Yu QW, Fang SQ, Zheng YK, Qi JC. MiR-650 inhibits the progression of glioma by targeting FAM83F. Eur Rev Med Pharmacol Sci. 2018;22:8391-8.

38. Yuan C, Xu L, Du P, Pang J. miRNA-650 exerts anti-leukemia activity by inhibiting cell proliferation through Gfi1 targeting. Tumori. 2018;104:369-74.

39. Dhayade S, Kaesler S, Sinnberg T, Dobrowinski H, Peters S, Naumann U, et al. Sildenafil potentiates a CGMP-dependent pathway to promote melanoma growth. Cell Rep. 2016;14:2599-610.

40. Yang L, Lei L, Zhao Q, Gong Y, Guan G, Huang S. C-Type Natriuretic peptide/natriuretic peptide receptor 2 is involved in cell proliferation and testosterone production in mouse Leydig cells. World J Mens Health. 2019; 37:186-98.

41. Chen WX, Liu HH, Li RX, Mammadov G, Wang JJ, Liu FF, et al. C-type natriuretic peptide stimulates osteoblastic proliferation and collagen-X expression but suppresses fibroblast growth factor-23 expression in vitro. Pediatr Rheumatol Online J. 2020;18:46.

\section{Publisher's Note}

Springer Nature remains neutral with regard to jurisdictional claims in published maps and institutional affiliations.

Ready to submit your research? Choose BMC and benefit from:

- fast, convenient online submission

- thorough peer review by experienced researchers in your field

- rapid publication on acceptance

- support for research data, including large and complex data types

- gold Open Access which fosters wider collaboration and increased citations

- maximum visibility for your research: over $100 \mathrm{M}$ website views per year

At $\mathrm{BMC}$, research is always in progress.

Learn more biomedcentral.com/submissions 